\title{
Carbon nanotube-graphene composite film as transparent conductive electrode for GaN-based light-emitting diodes
}

\author{
Chun Hong Kang,,$^{1,2}$ Chao Shen, ${ }^{3}$ M. Shuaib M. Saheed, ${ }^{2}$ Norani Muti \\ Mohamed, ${ }^{2}$ Tien Khee Ng, ${ }^{3}$ Boon S. Ooi, ${ }^{3}$ and Zainal Arif Burhanudin ${ }^{1,2, a)}$ \\ ${ }^{1}$ Department of Electrical and Electronic Engineering, Universiti Teknologi PETRONAS, 32610 Bandar Seri \\ Iskandar, Perak Darul Ridzuan, Malaysia \\ ${ }^{2}$ Center of Innovative Nanostructures and Nanodevices, Universiti Teknologi PETRONAS, 32610 Bandar Seri \\ Iskandar, Perak Darul Ridzuan, Malaysia \\ ${ }^{3}$ Photonics Laboratory, King Abdullah University of Science and Technology (KAUST), Thuwal 23955-6900, \\ Kingdom of Saudi Arabia
}

Transparent conductive electrodes (TCE) made of carbon nanotube (CNT) and graphene composite for GaN-based light emitting diodes (LED) are presented. The TCE with 533- $\Omega / \square$ sheet resistance and $88 \%$ transmittance were obtained when chemical-vapor-deposition (CVD) grown graphene was fused across CNT networks. With an additional 2-nm thin $\mathrm{NiO}_{\mathrm{x}}$ interlayer between the TCE and top $p$-GaN layer of the LED, the forward voltage was reduced to $5.12 \mathrm{~V}$ at $20-\mathrm{mA}$ injection current. Moreover, four-fold improvement in terms of light output power was also observed. The improvement can be ascribed to the enhanced lateral current spreading across the hybrid CNTgraphene TCE before injection into the p-GaN layer

\section{THE MANUSCRIPT}

Carbon-based materials, for example one-dimensional carbon nanotube (CNT) and two-dimensional graphene, have been of great interest in broad range of applications, such as field-emission devices ${ }^{1,2}$, sensors ${ }^{3,4}$, lithium-sulfur batteries $^{5,6}$, supercapacitors ${ }^{7}$ and field-effect transistors ${ }^{8}$. Both of the $\mathrm{sp}^{2}$-hybridized carbon materials possess superior thermal conductivity, mechanical robustness and high electrical conductivities. The fact that they are only several atomic layer thin make them highly transparent. They are viable alternatives for transparent conductive electrode (TCE) in place of indium tin oxide (ITO) that is known to have chemical instability, scarcity in supply and brittleness

\footnotetext{
a) Author to whom correspondence should be addressed. Electronic mail: zainabh@petronas.com.my
} 
unsuitable for flexible devices. ${ }^{910}$ Recently, graphene has been proposed and demonstrated as TCE on GaN-based LED. However, due to large work function $(\Phi)$ discrepancy with $p-\mathrm{GaN}^{11}$, high forward voltage ${ }^{12}$ and poor current spreading ${ }^{13}$, the performance of the LED with graphene TCE is still not at par with ITO-based TCE. Hence, in order to improve the performance of the graphene-based TCE on GaN-based LED, many groups have suggested a more exquisite solid-phase layer-stacking, hybridizing graphene-based TCE with ITO $\operatorname{rods}^{14}$, thin ITO layer ${ }^{15,16}$, metal nanowires (i.e. $\mathrm{Ag}^{17,18}, \mathrm{Cu}^{19} \mathrm{NWs}$ ) or Au nanoparticle (NPs) ${ }^{9,20}$. As for CNT-based TCE on LED, Jung et al. has demonstrated that using dip-coated CNT stacked with ITO layer, a more uniform current spreading and up to $60 \%$ higher output power compared to ITO-based TCE could be achieved. ${ }^{21}$ Note that although significant efforts have been made to improve the performance of carbon-based TCE, most of the reported TCE schemes still require the scarce indium (In).

Carbon, on the other hand, is abundant in nature. TCE derived from carbon are also relatively easy to produce owing to the scalability of chemical vapor deposition (CVD) techniques. ${ }^{3}$ However, stand-alone randomly dispersed network of CNT exhibits poor conductivity. Kim et al., for example, reported an inhomogeneous current flow on highly porous spin-coated film of CNT network. ${ }^{22}$ Similarly, graphene typically has discontinuities, wrinkles and boundaries that affects its extrinsic properties. ${ }^{8}$ Reduction of charge carrier mobility has been observed for dopedgraphene layer on GaN-based LED and the improvement is deemed rather unstable. ${ }^{11,23}$ Such inadequacies limit carrier transport and subsequently degrade the performance of the actual devices.

In an effort to overcome the inadequacies, a composite material made of 1D CNT and 2D graphene has been proposed. It can be prepared by techniques such as electrophoretic deposition (EPD) $)^{24}$, solution processing ${ }^{7,25}$ and chemical vapor deposition $(\mathrm{CVD})^{5,6}$. The composite material has been reported to have an enhanced electrical conductivity that can be explained by (a) CNT act as conducting bridge across grain boundaries of graphene and (b) graphene layer provides conducting pathway across the separated CNT strands. ${ }^{8,22,26}$ Inspired by these enhanced performance, herein, we presented TCE made of CNT-graphene composite for GaN-based LED. The composite film has relatively low average sheet resistance $(\sim 533 \Omega / \square)$ and high optical transmittance $(\sim 88 \%)$. The fabricated LED showed lower forward voltage of $5.12 \mathrm{~V}$ and four-fold improved light output power when a 2-nm thin $\mathrm{NiO}_{\mathrm{x}}$ is placed in between CNT-graphene composite layer and the top $p$-GaN layer. This work explored a pathway towards realizing a truly ITO-free carbon-based TCE for high performance GaN-based LEDs. 
The key processes and device structure are summarized in Fig. 1. As a start, highly purified CVD-synthesized CNT powder was dispersed in 1,2-dichlorobenzene $\left(\mathrm{C}_{6} \mathrm{H}_{4} \mathrm{Cl}_{2}\right)$ solution. The solution was then spin-coated onto copper (Cu) foil (see Fig. 1(a)). This allows CNT films to be formed on the $\mathrm{Cu}$ foil. The film together with the foil were then placed in a CVD system. At $1000{ }^{\circ} \mathrm{C}$, a mixture of methane $\left(\mathrm{CH}_{4}\right)$, argon $(\mathrm{Ar})$ and hydrogen $\left(\mathrm{H}_{2}\right)$ gases were released into the CVD system. At this temperature, methane would break down, forming a layer of carbon atoms, also known as graphene, across the CNT film (see Fig. 1(b)). The CNT-graphene composite film was then spin-coated with polymethyl methacrylate (PMMA), followed by immersion into iron trichloride $\left(\mathrm{FeCl}_{3}\right)$ solution to etch the underlying $\mathrm{Cu}$ foil (see Fig. 1(c)). After etching, the CNT-graphene composite film was cleaned in diluted hydrochloric acid (HCl) to remove $\mathrm{Cu}$ residues and subsequently washed repeatedly with deionized (DI) water. The cleaned PMMA/CNTgraphene composite film was then scooped out with a commercially available 'blue LED structure' that essentially consists of $c$-plane sapphire, layered with $0.7 \mu \mathrm{m}$ undoped-GaN, $2.7 \mu \mathrm{m} \mathrm{Si-doped} n$-GaN, six-period of InGaN/GaN ( $3 \mathrm{~nm} / 10 \mathrm{~nm}$ ) multiple-quantum-well (MQW) active region, and $270 \mathrm{~nm} \mathrm{Mg-doped} p$-GaN top most layer. To improve contact adhesion and remove trapped water, the composite film and the blue LED structure were then baked twice at $80^{\circ} \mathrm{C}$ and $130^{\circ} \mathrm{C}$ respectively. PMMA was then removed by soaking the whole structure into acetone (Fig. 1(e)). After the synthesis and transfer of CNT-graphene composite film onto the blue LED structure, the mesa structures were defined using inductively-coupled plasma reactive ion etcher (ICP-RIE). Initially, unwanted region of the composite film was removed using a mixture of argon and oxygen plasma. Then, subsequent GaN layers were removed by standard $\mathrm{Cl}_{2}$-based dry etch process until the $n$-doped $\mathrm{GaN}$ layer was exposed. A 40 -nm thin $\mathrm{SiO}_{2}$ layer was then deposited to act as insulating layer between $p$-metal pad and other GaN layers. Finally, a thin 10-nm Ti adhesion layer and 100-nm Au contact layer were deposited using e-beam evaporator to form the contact pads. The final fabricated circular mesa structure with chip area of $200 \mu \mathrm{m}$ in diameter is shown in Fig. 1(f).

Figure 2 shows the surface morphologies of bare CNT, graphene and CNT-graphene composite film, with their corresponding selected area electron diffraction (SAED) patterns. As shown in Fig. 2 (a), CNTs used in our experiment were purified multi-walled CNT (MWCNT) with average outer diameter of $\sim 10 \mathrm{~nm}$ and consisted of about 5 outer layers. Inset of Fig. 2(a) shows the corresponding SAED pattern of (002) planes of hexagonal graphitic lattice, typically observed in a single strand of $\mathrm{CNT}^{27}$ Figure 2 (b) shows the high resolution transmission electron microscopy (HRTEM) image of bare graphene film. The SAED pattern as in the inset of Fig. 2(b) shows clear six- 
fold symmetry hexagonal pattern indicating that single layer ${ }^{15,28}$, or at most double layer high quality graphene has been synthesized. Using the same recipe, similar high quality graphene layer could be expected for the CNT-graphene composite film. Figure 2 (c) shows field emission scanning electron microscope (FESEM) image of randomly dispersed CNT network with graphene as intertube 'filler' layer (not visible under FESEM). Due to the random dispersion of CNT, the formation of CNT network consisted of 2-4 inter-stacked layers and partial CNT bundles. The density of CNT were measured to be approximately $10^{2} \mu \mathrm{m}^{2}$. Unlike metal NWs, CNTs are more susceptible to entanglement due their strong van der Waals interaction. Therefore, proper separation of individual CNTs and control over the desired density need to be done in order to obtain an optimized TCE performance. The presence of graphene surrounding separated network of CNTs were confirmed from HRTEM images (see Fig. 2 (d)). With graphene interlinking the sidewalls of CNTs, better charge transport across the composite film is expected. Note that although the dispersed CNT was exposed to a relatively high temperature at $\sim 1000{ }^{\circ} \mathrm{C}$ during the $\mathrm{CVD}$ process, no significant structural defects were observed either from HRTEM images or Raman spectrum (discussed in the following paragraph). This extremely high thermal stability of CNTs is expected as there is report showing CNTs can withstand temperature even up to $\sim 2000{ }^{\circ} \mathrm{C}$ before collapsing and breaking..$^{29}$ Moreover, the corresponding SAED pattern of CNT-graphene composite film as in Fig. 2 (e), shows that the ring patterns of (002) graphitic lattice originating from CNTs are still present. This signifies that the overall crystallinity of the materials is properly maintained. ${ }^{27}$

Once the crystallinity of the CNT-graphene composite film has been ascertained, it is transferred onto the $p$-GaN layer of the blue LED structure. Surface roughness of the composite film on the blue LED structure are initially measured using atomic force microscope (AFM) (see Fig. 3(a)). It exhibits an average surface roughness (Ra) of $\sim 11.8$ nm. A height profile scan across line 1 in Fig. 3 (a) shows a maximum of $\sim 31 \mathrm{~nm}$ disparity, indicating only about 3 stacks of CNT networks are present. Such surface profile is acceptable for LED fabrication processing. ${ }^{17}$ Once the LED has been fabricated, the presence of CNT-graphene composite film was confirmed by Raman spectra using 532 nm laser excitation source. Typical Raman spectra for such samples at point A, B and C are shown in Fig. 3 (b). A strong $G$ peak $\left(\mathrm{G}^{+}\right.$and $\left.\mathrm{G}^{-}\right)$near $\sim 1585 \mathrm{~cm}^{-1}$ and $2 \mathrm{D}$ peak near $\sim 2670 \mathrm{~cm}^{-1}$, indicates the presence of the composite film on GaN-based LEDs at these points of interest. The co-existence of CNT and graphene is represented by a single Lorentzian feature at 2D peak near $\sim 2670 \mathrm{~cm}^{-1}$, while two broader peaks, $\mathrm{G}^{+}$near $\sim 1589 \mathrm{~cm}^{-1}$ and $\mathrm{G}^{-}$near $\sim 1580 \mathrm{~cm}^{-}$ ${ }^{1}$ were observed from the graphitic-related $\mathrm{G}$ peak due to the displacement of carbon atoms along the circumferential directions of the $\mathrm{CNT}^{8}{ }^{8}$ The other two $\mathrm{E}_{2}\left(\sim 570 \mathrm{~cm}^{-1}\right)$ and $\mathrm{A}_{1}\left(\sim 735 \mathrm{~cm}^{-1}\right)$ peaks simply originated from strained GaN 
layers. It should be noted that no significant defect-related D peak is present, indicating high degree of crystallinity and minimal defect density from the composite film. In contrast to other solution-based processes, which typically induce higher topological and structural defects, CVD-based preparation method is more robust and less damaging to the intrinsic structures of the materials. ${ }^{1,25}$ This is further supported by the small variation in the $\mathrm{I}_{\mathrm{D}} / \mathrm{I}_{\mathrm{G}}$ ratio of CNTgraphene composite film, which were calculated to be in between 0.05 to 0.10 (see Fig. 3 (b)). The small increment of $\mathrm{I}_{\mathrm{D}} / \mathrm{I}_{\mathrm{G}}$ ratio in CNT-graphene composite film indicates that low impurity level and high crystallinity even after standard LED fabrication processes has been carried out.

Next, the fabricated LED chips were then characterized using a customized prober that is attached to a Keithley 2400 source meter and Newport 2936-C optical power meter. Figure 4 (a) shows the current-voltage (I-V) characteristics of the LEDs under direct current (DC) injection for three different TCE schemes, i.e. graphene, CNTgraphene and CNT-graphene with thin $\mathrm{NiO}_{\mathrm{x}}$ interlayer. At a typical 20-mA current injection, LED with bare graphene TCE demonstrated a relatively high forward voltage, $\mathrm{V}_{f}=6.93 \mathrm{~V}$, that is consistent with other reported works. ${ }^{913,15,30}$ This high $\mathrm{V}_{f}$ is primarily attributed to the large discrepancy of Schottky barrier height/work function mismatch between graphene $\left(\Phi_{\mathrm{G}}=4.5 \mathrm{eV}\right)$ and $p-\mathrm{GaN}\left(\Phi_{p-\mathrm{GaN}}=7.5 \mathrm{eV}\right){ }^{20,30}$ Apart from that, unfavourable conductivity and charge scattering across grain boundaries of polycrystalline graphene film also affected the lateral current spreading performance of bare graphene TCE. ${ }^{12}$ On the other hand, when CNT-graphene composite film was adopted as TCE, the $\mathrm{V}_{f}$ was reduced down to $6.12 \mathrm{~V}$ at $20 \mathrm{~mA}$. This is largely due to improved interconnected network and reduced contact resistivity of network CNT-graphene composite film. Taking advantage of intrinsic graphitic affinity of both $\mathrm{sp}^{2}$-hybridized CNT and graphene, the carbon framework based on $\pi$-electronic interaction facilitates symmetrical carriers transport and lower intertube resistance across percolated CNT network through extra transport channel formed by graphene layer., ${ }^{1,5,731}$ At the same time, the framework retains the extremely high carrier mobility in individual chain of CNTs. In the future, we anticipate that a much higher conductivity can be achieved in the hybridized carbon structures when the composition ratio of CNT/graphene is optimized using a prediction and optimization model like the one recently proposed by Shim et al. ${ }^{26} \mathrm{~A}$ second look at the $I-V$ curves also shows that the fabricated LED has high turn-on voltage which could be due to poor hole injection into the $p$-GaN resulting from work function mismatch of both carbon nanostructures $\left(\Phi_{\mathrm{CNT}}=4.5\right.$ to $4.8 \mathrm{eV}$ and $\left.\Phi_{\mathrm{G}}=4.5 \mathrm{eV}\right)$. In order to overcome this, a 2-nm thin layer of $\mathrm{NiO}_{\mathrm{x}}\left(\Phi_{\mathrm{NiOx}}=\sim 5.1 \mathrm{eV}\right)$ was inserted as interlayer between $p$-GaN and CNT-graphene composite film. As a result, the fabricated CNT-graphene composite film with thin $\mathrm{NiO}_{\mathrm{x}}$ interlayer demonstrated a much 
improved $\mathrm{V}_{f}$ of $5.12 \mathrm{~V}$ at $20 \mathrm{~mA}$. Such reduction of $\mathrm{V}_{f}$ that is attributed to the lowering of Schottky barrier height using $\mathrm{NiO}_{\mathrm{x}}$ interlayer has been reported previously on graphene-based TCE. ${ }^{12,13}$ By using the combined strategies of CNT-graphene composite films and $\mathrm{NiO}_{\mathrm{x}}$ interlayer, the achieved $\mathrm{V}_{f}$ at $20 \mathrm{~mA}$ on GaN-based LEDs is comparable to other proposed TCE scheme such as $3 \mathrm{~nm}$ ITO +3 layer graphene $\left(\mathrm{V}_{f}=5.6 \mathrm{~V}\right)^{15}$, graphene/Ag NWs $\left(\mathrm{V}_{f}=6.6 \mathrm{~V}\right)^{18}$, $\mathrm{HNO}_{3}$-doped graphene $\left(\mathrm{V}_{f}=5.35 \mathrm{~V}\right)^{30}$ and Au/graphene $\left(\mathrm{V}_{f}=4.63 \mathrm{~V}\right)^{9}$.

Light output power versus injected current $(L-I)$ characteristics for LEDs with the three TCE schemes are shown in Fig. 4 (b). The light output power (LOP) of the LEDs was collected from the top of the device through a $5 \times$ objective lens of an optical microscope. All devices have an emission peak at $~ 452 \mathrm{~nm}$. From Fig. 4(b), the light output power of the LEDs at 4-mA injection current for graphene, CNT-graphene and CNT-graphene with $\mathrm{NiO}_{\mathrm{x}}$ interlayer TCEs are $0.52,1.30$ and $2.08 \mathrm{~mW}$, respectively. The compelling four-fold LOP improvement of CNT-graphene composite film is most likely due to enhanced current transport across the in-plane structure of composite film and higher current injection into $p-\mathrm{GaN}$ via $\mathrm{NiO}_{\mathrm{x}}$ interlayer.

In order to establish the physical parameters related to the improvement, four-point probe measurement, Halleffect mobility measurement and UV-VIS transmission spectroscopy were carried out. Figure 4 (c) shows the sheet resistance $\left(R_{s}\right)$ obtained from the four-point probe measurement for graphene, stacked graphene/CNT, stacked CNT/graphene and CNT-graphene composite films on oxidized Si substrates. An average $R_{s}$ of $1122 \Omega / \square$ was obtained from standard CVD-grown graphene. As for solid-phase stacked CNT/graphene or stacked graphene/CNT, the $R_{s}$ $\sim 1110 \Omega / \square$ is apparently close to the $R_{S}$ of bare graphene. This observation is consistent with works reported elsewhere. ${ }^{8,32}$ CNT-graphene composite films, however, exhibits a much lower average $R_{s}$ of $533 \Omega / \square$. Such drop in $R_{S}$ is likely due to the direct CVD-growth of graphene across the network of CNT that improves the CNT/graphene junction contacts and facilitates charge transport. ${ }^{5-7,26}$ Next, room-temperature Hall-effect mobility of these thin films were measured using van der Pauw method with silver (Ag) paste on a four-point contacts. As shown in Fig. 4 (c), graphene and both solid-phase stacked layer of CNT/graphene and graphene/CNT exhibit low average mobility. CNTgraphene composite film, on the other hand, shows its superiority with an average mobility of $\sim 1562 \mathrm{~cm}^{2} / \mathrm{V}$-s. This $\sim 50 \%$ improvement in both mobility and sheet resistance definitely contributes to the four-fold LOP improvement observed earlier. Finally, the optical transmission of each layer, i.e. CNT-graphene, graphene, $\mathrm{NiO}_{\mathrm{x}}$ and ITO film, measured using UV-VIS spectroscopy are shown in Fig. 4 (d). Throughout the near ultra-violet to visible wavelength, 
graphene and $\mathrm{NiO}_{\mathrm{x}}$ appear to be the most transparent with transmission in between $95-100 \%$. CNT-graphene composite is about $10 \%$ less transparent than graphene and $\mathrm{NiO}_{\mathrm{x}}$, while ITO is consistently low reaching $55 \%$ at 400 nm near-UV wavelength. More interestingly is the inset of Fig. 4 (d) that shows the optical transmission of the targeted 450-nm wavelength of the blue GaN LED. The inset shows the transmission of CNT-graphene, graphene, $\mathrm{NiO}_{\mathrm{x}}$ and ITO were 88, 96, 97 and 75\%, respectively. Note that although CNT-graphene has slightly lower transparency than graphene and $\mathrm{NiO}_{\times}$, it has the lowest $R_{S}$ and highest mobility. Hence, based on $I-V$ and $L-I$ curves of the fabricated LED, it can be argued that for high LOP, lateral electrical conductivity of TCEs is still of utmost importance as compared to their optical transparency. Similar arguments was also reported in Ag NW/graphene-based TCEs. ${ }^{18}$ Therefore, the higher LOP observed from CNT-graphene composite can be attributed to the improved lateral sheet conductivities that provides more uniform current spreading across the TCE layer before injection into $p$-GaN layer.

In summary, the performance of CNT-graphene composite film as transparent conductive electrode (TCE) for GaN-based LED has been systematically investigated. By employing the composite film with thin $\mathrm{NiO}_{\mathrm{x}}$ interlayer, the forward voltage of the device at 20 -mA injection current is significantly reduced to $5.12 \mathrm{~V}$. Despite the low $\sim 88 \%$ transparency of the composite film, four-fold improvement in terms of light output power was obtained by using the proposed scheme. The improvement is primarily due to the enhanced electrical pathway in the TCE by conducting graphene 'filler' layer through the CNT network, leading to the reduction in sheet resistance and promoting superior current transport across the interconnected all-carbon network. With great flexibility, high thermal resistance and optically transparent near the UV region, it is anticipated that the demonstrated carbon-based composite to be the TCE of choice not only for high performance GaN-based LEDs but also for future flexible light emitting devices.

The authors would like to acknowledge the Graduate Assistantship Scheme from Universiti Teknologi PETRONAS. This work was partially supported by the Fundamental Research Grant Scheme from Ministry of Higher Education of Malaysia (Grant No. FRGS/1/2015/TK04/UTP/02/2), KAUST Baseline Funding (Grant No. BAS/1/1614-01-01), and King Abdulaziz City for Science and Technology (KACST) Technology Innovation Center (TIC) for Solid State Lighting (Grant No. KACST TIC R2-FP-008).

\footnotetext{
${ }^{1}$ M. Song, P. Xu, Y. Song, X. Wang, Z. Li, X. Shang, H. Wu, P. Zhao, and M. Wang, AIP Adv. 5, 097130 (2015).

${ }^{2}$ R. Khare, D. B. Shinde, S. Bansode, M. A. More, M. Majumder, V. K. Pillai, and D. J. Late, Appl. Phys. Lett. 106, 023111 (2015).

${ }^{3}$ M. S. M. Saheed, N. Muti Mohamed, and Z. Arif Burhanudin, Appl. Phys. Lett. 104, 123105 (2014).

${ }^{4}$ L. Shahriary, H. Ghourchian, and A. A. Athawale, J. Nanotechnol. 2014, 10 (2014).

${ }^{5}$ M.-Q. Zhao, X.-F. Liu, Q. Zhang, G.-L. Tian, J.-Q. Huang, W. Zhu, and F. Wei, ACS Nano 6, 10759 (2012).
} 
${ }^{6}$ H.-J. Peng, J.-Q. Huang, M.-Q. Zhao, Q. Zhang, X.-B. Cheng, X.-Y. Liu, W.-Z. Qian, and F. Wei, Adv. Funct. Mater. 24, 2772 (2014).

${ }^{7}$ L. Peng, Y. Feng, P. Lv, D. Lei, Y. Shen, Y. Li, and W. Feng, J. Phys. Chem. C 116, 4970 (2012).

${ }^{8}$ S. H. Kim, W. Song, M. W. Jung, M.-A. Kang, K. Kim, S.-J. Chang, S. S. Lee, J. Lim, J. Hwang, S. Myung, and K.-S. An, Adv. Mater. 26, 4247 (2014).

${ }^{9}$ S. Chandramohan, J. Hye Kang, Y. S. Katharria, N. Han, Y. Seon Beak, K. Bok Ko, J. Bae Park, H. Kyu Kim, E.K. Suh, and C.-H. Hong, Appl. Phys. Lett. 100, 023502 (2012).

${ }^{10}$ S. H. Chae and Y. H. Lee, Nano Convergence 1, 1 (2014).

${ }^{11}$ L. Wang, Y. Cheng, Z. Liu, X. Yi, H. Zhu, and G. Wang, ACS Appl. Mater. Interfaces 8, 1176 (2016).

${ }^{12}$ S. Chandramohan, K. Bok Ko, J. Han Yang, B. Deul Ryu, Y. S. Katharria, T. Yong Kim, B. Jin Cho, and C.-H. Hong, J. Appl. Phys. 115, 054503 (2014).

13 J.-P. Shim, T. Hoon Seo, J.-H. Min, C. Mo Kang, E.-K. Suh, and D.-S. Lee, Appl. Phys. Lett. 102, 151115 (2013).

${ }^{14}$ M. J. Yun, H.-D. Kim, K. H. Kim, H. J. Sung, S. Y. Park, H.-M. An, and T. G. Kim, Thin Solid Films 547, 95 (2013).

${ }^{15}$ X. Kun, X. Chen, D. Jun, Z. Yanxu, G. Weiling, M. Mingming, Z. Lei, and S. Jie, Appl. Phys. Lett. 102, 162102 (2013).

${ }^{16}$ W.-C. Lai, C.-N. Lin, Y.-C. Lai, P. Yu, G. C. Chi, and S.-J. Chang, Opt. Express 22, A396 (2014).

${ }^{17}$ G.-J. Jeong, J.-H. Lee, S.-H. Han, W.-Y. Jin, J.-W. Kang, and S.-N. Lee, Appl. Phys. Lett. 106, 031118 (2015).

18 Z. Li, J. Kang, Z. Liu, C. Du, X. Lee, X. Li, L. Wang, X. Yi, H. Zhu, and G. Wang, AIP Adv. 3, 042134 (2013).

${ }^{19}$ H. Guo, N. Lin, Y. Chen, Z. Wang, Q. Xie, T. Zheng, N. Gao, S. Li, J. Kang, D. Cai, and D.-L. Peng, Sci. Rep. 3, 2323 (2013).

${ }^{20}$ M. Choe, C.-Y. Cho, J.-P. Shim, W. Park, S. K. Lim, W.-K. Hong, B. Hun Lee, D.-S. Lee, S.-J. Park, and T. Lee, Appl. Phys. Lett. 101, 031115 (2012).

${ }^{21}$ S.-Y. Jung, K. H. Kim, S.-Y. Jeong, J.-W. Jeon, J. Moon, S. Y. Lee, J.-O. Song, Y. T. Byun, and T.-Y. Seong, Electrochem. Solid State Lett. 13, H33 (2010).

${ }^{22}$ M. Kim and Y. C. Kim, Synthetic Met. 198, 31 (2014).

${ }^{23}$ H. Kim, H. H. Kim, J. I. Jang, S. K. Lee, G.-W. Lee, J. T. Han, and K. Cho, Adv. Mater. 26, 8141 (2014).

${ }^{24}$ M. D. Lima, M. J. de Andrade, C. P. Bergmann, and S. Roth, J. Mater. Chem. 18, 776 (2008).

${ }^{25}$ Y. Jia, K. Yu, and K. Qian, Nanoscale Res. Lett. 8, 1 (2013).

${ }^{26}$ W. Shim, Y. Kwon, S.-Y. Jeon, and W.-R. Yu, Sci. Rep. 5, 16568 (2015).

27 Y. C. Jeong, K. Lee, T. Kim, J. H. Kim, J. Park, Y. S. Cho, S. J. Yang, and C. R. Park, J. Mater. Chem. A 4, 819 (2016).

${ }^{28}$ J. C. Meyer, A. K. Geim, M. I. Katsnelson, K. S. Novoselov, T. J. Booth, and S. Roth, Nature 446, 60 (2007).

${ }^{29}$ K. M. Liew, C. H. Wong, X. Q. He, and M. J. Tan, Phys. Rev. B 71, 075424 (2005).

${ }^{30}$ Y. Doo-Hyeb, Y. Young-Jun, C. HongKyw, K. Suck-Hwan, C. Sung-Yool, and C. Choon-Gi, Nanotechnology 24, 075202 (2013).

${ }^{31}$ B. Liu, C. Li, Q.-L. Liu, J. Dong, C.-W. Guo, H. Wu, H.-Y. Zhou, X.-J. Fan, X. Guo, C. Wang, X.-M. Sun, Y.-H. Jin, Q.-Q. Li, and S.-S. Fan, Appl. Phys. Lett. 106, 033101 (2015).

${ }^{32}$ I. N. Kholmanov, C. W. Magnuson, R. Piner, J.-Y. Kim, A. E. Aliev, C. Tan, T. Y. Kim, A. A. Zakhidov, G. Sberveglieri, R. H. Baughman, and R. S. Ruoff, Adv. Mater. 27, 3053 (2015). 


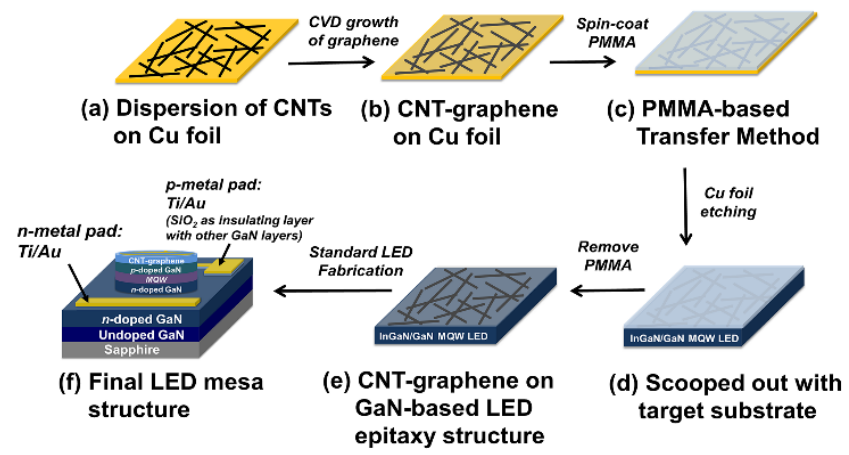

FIG. 1. A schematic illustrating the procedures involved in $(a, b)$ synthesis and (c-e) transfer of CNT-graphene composite film onto commercially available GaN-based LED epitaxy structure and lastly (f) the final LED mesa structure. The CNT-graphene composite film which acts as TCE sits on top of a 2-nm thin $\mathrm{NiO}_{\mathrm{x}}$ layer not shown in the schematic.
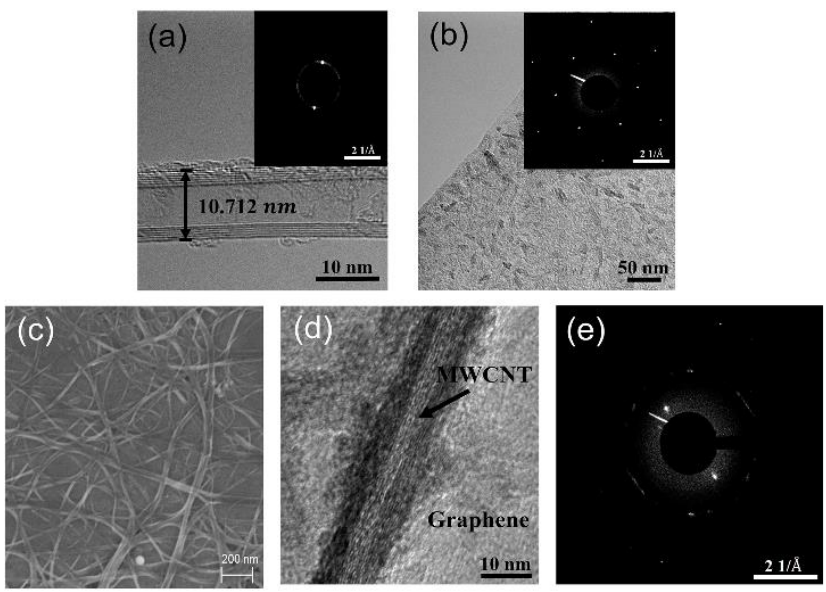

FIG. 2. HRTEM images of (a) single strand of MWCNT and their corresponding SAED pattern (inset). (b) Bare graphene film and their corresponding SAED pattern (inset). (c) Top-view FESEM image of CNT-graphene composite film on GaN-based LED. (d) HRTEM image of coexistence of CNT and graphene on the composite film. (e) SAED pattern of CNT-graphene composite film. 

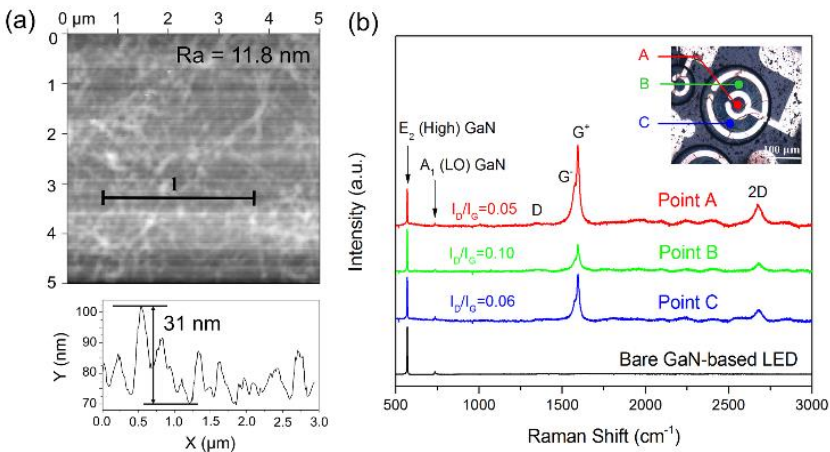

FIG. 3. (a) AFM image of CNT-graphene composite film on GaN-based LED before fabrication, with scan area of $5 \times 5 \mu$ m. (b) Raman spectra of CNT-graphene composite film on fabricated GaN-based LED.
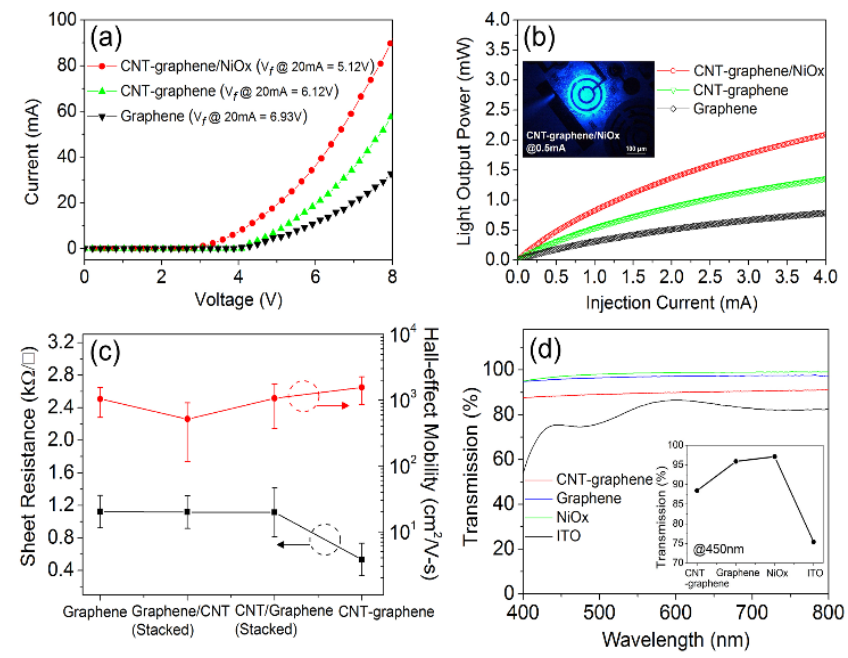

FIG. 4. (a) Current-Voltage (I-V) and (b) Light output power-Current $(L-I)$ characteristics of graphene, CNT-graphene and CNT-graphene composite film with $\mathrm{NiO}_{\mathrm{x}}$ interlayer as TCE for the $200 \mu \mathrm{m}$ diameter LEDs. Inset in (b) shows the optical micrograph image of a working LED based on CNT-graphene composite film. (c) Sheet resistance and Halleffect mobility of graphene, stacked graphene (top layer)/CNT (bottom layer), CNT (top layer)/graphene (bottom layer) and CNT-graphene composite films on oxidized Si substrates. (d) Optical transmission of CNT-graphene, graphene, $\mathrm{NiO}_{\mathrm{x}}$ and conventional ITO film throughout visible wavelength region. Inset graph in (d) shows the percentage of transmission at target wavelength $(\sim 450 \mathrm{~nm})$ of fabricated blue LEDs. 